\title{
Case Report \\ The Value of Desmethylclozapine and Serum CRP in Clozapine Toxicity: A Case Report
}

\author{
Khalid Abou Farha, ${ }^{1}$ Andre van Vliet, ${ }^{2}$ Henderikus Knegtering, ${ }^{3,4}$ and Richard Bruggeman ${ }^{4}$ \\ ${ }^{1}$ QPS Netherlands B.V. 9713 GZ Groningen, The Netherlands \\ ${ }^{2}$ PRA International, 9471 GP Zuidlaren, The Netherlands \\ ${ }^{3}$ Lentis Mental Health Organisation, 9725 AG Groningen, The Netherlands \\ ${ }^{4}$ Rob Giel Onderzoekscentrum, Department of Psychiatry, University Medical Centre Groningen, University of Groningen, \\ 9700 RB Groningen, The Netherlands
}

Correspondence should be addressed to Khalid Abou Farha, khalid.abou.farha@qps.com

Received 17 June 2012; Accepted 3 July 2012

Academic Editors: C. Lançon and F. Pillmann

Copyright (C) 2012 Khalid Abou Farha et al. This is an open access article distributed under the Creative Commons Attribution License, which permits unrestricted use, distribution, and reproduction in any medium, provided the original work is properly cited.

\begin{abstract}
Clozapine, an atypical antipsychotic, has proved to be superior to other antipsychotics in treating patients with refractory schizophrenia. An increased plasma clozapine level above the therapeutic window may be associated with serious adverse events including paralytic ileus. Clozapine toxicity may occur in association with infection or after drug overdose. In a medical emergency situation, differentiating between a toxic clozapine ingestion and an infection-induced toxicity might be hindered by associated CNS changes and by the clozapine modulation of the inflammatory process. This may delay prompt initiation of a tailored treatment strategy. Here, we report a case of paralytic ileus developed within the context of clozapine toxicity. Although the underlying cause of toxicity was not clinically obvious, giving antimicrobial therapy resulted in an improvement in the patient's clinical condition. This report indicates the value of serum levels of C-reactive protein and desmethylclozapine, major metabolite of clozapine, in the treatment of aetiologically unclear clozapine toxicity.
\end{abstract}

\section{Introduction}

Clozapine (CLZ), an atypical antipsychotic, has proved to be superior to other antipsychotics in treating patients with refractory schizophrenia [1]. Nevertheless, clozapine use might be limited by a number of serious adverse events including paralytic ileus, a CLZ-induced anticholinergic effect [2]. Paralytic ileus associated with clozapine treatment might be infection-induced $[3,4]$ or due to a toxic clozapine ingestion (overdose). Differential diagnosis can be challenging to the treating physician given the associated CNS changes, such as confusion, sedation delirium or even coma, and by the clozapine modulation of the inflammatory process [5-10] that may mask clinical signs and laboratory markers of inflammation. In this report, we discuss the value of serum CRP and the CLZ metabolite, desmethylclozapine, in the differential diagnosis and treatment of aetiologically unclear clozapine toxicity.

\section{Case Presentation}

A schizophrenic patient, male, 44 years old, was referred to the emergency room because of symptoms of dyspnoea, coughing, abdominal pain, nausea, vomiting, diarrhea, and somnolence. The gastrointestinal symptoms began a few days before his admission. The patient is a nonsmoker and has no similar occurrences in his medical history. His outpatient medications included lithium (1000 mg/day), CLZ (300 mg/day), and omeprazole $(20 \mathrm{mg} /$ day) for gastrointestinal symptoms. He had been taking CLZ for more than 10 years. The patient was admitted to the gastrointestinal surgery ward. On admission, a physical examination revealed a respiratory rate of 24 breaths/minute, blood pressure $135 / 100 \mathrm{mmHg}$, high pulse rate $(125 \mathrm{bpm})$, normal body temperature of $37.1^{\circ} \mathrm{C}$, oxygen saturation of $94 \%$, diminished vesicular breath sounds on pulmonary auscultation, abdominal distension, and tenderness, hypertympanic note 
on abdominal percussion, and hypoactive intestinal sounds. Laboratory tests revealed an elevated CRP (130 mg/L), a normal leucocyte count of $9.5 \times 10^{9} / \mathrm{L}$, normal polymorph nuclear neutrophil $(\mathrm{PMN})$ count of $7.5 \times 10^{9} / \mathrm{L}(<80 \%$ of total leucocyte count) with no left shift, serum ASAT $107 \mathrm{U} / \mathrm{L}$ (reference value $<40 \mathrm{U} / \mathrm{L}$ ), serum ALAT $223 \mathrm{U} / \mathrm{L}$ (reference value $<45 \mathrm{U} / \mathrm{L}$ ), serum creatinine $173 \mathrm{umol} / \mathrm{L}$ (reference range $70-110 \mathrm{umol} / \mathrm{L}$ ), plasma glucose $14.3 \mathrm{mmol} / \mathrm{L}$ (reference range $3.5-7.8 \mathrm{mmol} / \mathrm{L})$, and serum sodium $(\mathrm{Na})$ level of $134 \mathrm{mmol} / \mathrm{L}$ (reference range $135-145 \mathrm{mmol} / \mathrm{L}$ ). A plain X-ray of the abdomen revealed dilated small bowel loops. Nonobstructive dilatation of the small intestine and transverse colon was confirmed on CT scan. A thorax X-ray revealed no pulmonary infiltrate. Determination of the plasma level of the antipsychotics revealed a CLZ level of $1301 \mu \mathrm{g} / \mathrm{L}$ (reference therapeutic range 200-600 ug/L), desmethylclozapine (Norclozapine, NCLZ) level of $515 \mu \mathrm{g} / \mathrm{L}$ making an NCLZ:CLZ ratio of approximately $40 \%$. The lithium level was $0.56 \mathrm{mmol} / \mathrm{L}$ (reference therapeutic range $0.6-0.8 \mathrm{mmol} / \mathrm{L}$ ). A diagnosis of paralytic ileus secondary to clozapine intoxication was made. CLZ and lithium were immediately discontinued. The patient received an i.v. nutritional supplement including NaCL $0.9 \%$, potassium chloride to compensate for the diarrhoea-induced potassium loss and gastrointestinal decompression using nasogastric tube. Because of a suspected pneumonia, amoxicillin/clavulanic acid i.v. at a dose of $200 \mathrm{mg}$ TID was administered for 10 days. Within the first week after institution of therapy and discontinuation of the antipsychotics, there was simultaneous improvement in the patient's condition, return of a normal gastrointestinal function, and a decline in CRP to $<5 \mathrm{mg} / \mathrm{L}$ and CLZ plasma level to $103 \mu \mathrm{g} / \mathrm{L}$ with an NCLZ level of $110 \mu \mathrm{g} / \mathrm{L}$ making an NCLZ:CLZ ratio of $107 \%$. Given that, lithium ( $400 \mathrm{mg} /$ day) and CLZ (50 mg/day) were readministered on day 9 postadmission (8th day of amoxicillin/clavulanic acid administration). Under CLZ readministration, the patient developed progressive watery diarrhoea and abdominal pain. Physical examination revealed abdominal tenderness, reduced intestinal peristaltic sounds, normal body temperature of $36.5^{\circ} \mathrm{C}$, blood pressure of $120 / 80 \mathrm{mmHg}$, and a pulse rate of $104 \mathrm{bpm}$. Serum levels of liver transaminases were within normal range (ASAT, $24 \mathrm{U} / \mathrm{L}$ ) or mildly elevated (ALAT, $76 \mathrm{U} / \mathrm{L}$ ). Similarly, serum alkaline phosphatase (ALP) was not elevated, $77 \mathrm{U} / \mathrm{L}$ (reference value $<120 \mathrm{U} / \mathrm{L}$ ). Although the leucocyte count was normal $(4.6 \times 10 \mathrm{E} 9 / \mathrm{L})$, there was reelevation in CRP $(87 \mathrm{mg} / \mathrm{L})$ and an increase in CLZ $(336 \mu \mathrm{g} / \mathrm{L})$ with an NCLZ level of $71 \mu \mathrm{g} / \mathrm{L}$, making an NCLZ:CLZ ratio of approximately $21 \%$. Faecal culture excluded the presence of pathogenic staphylococci, salmonella enteridis, and clostridia. Given that the onset of diarrhoea was simultaneous with readministration of CLZ, the CLZ was again discontinued on day 13 and a conservative approach adopted until the patient's condition improved 2 days later (i.e., day 15 postadmission). After complete stabilization of the somatic condition and psychiatric profile the patient was discharged with the following medications: risperidone depot $25 \mathrm{mg} / 2$ weeks, lithium $800 \mathrm{mg} /$ day and paroxetine $10 \mathrm{mg} /$ day. Figures 1 and 2 demonstrate the changes in CLZ,
NCLZ and CRP levels and serum transaminases during the course of the patient's illness and hospital admission.

\section{Discussion}

In this report, our patient demonstrated, on admission, a toxic high plasma CLZ level accompanied by paralytic ileus and an elevated CRP. There was no conclusive clinical, radiological or laboratory evidence of an infection-induced inflammatory response, such as fever, lung neutrophil infiltration, leucocytosis/neutrophilia, or PMN left shift. Such clinical presentation might lead to uncertainty as to the underlying cause of high CLZ, overdose versus infection-induced, and hence in selecting a treatment strategy including early administration of antimicrobial therapy. Nevertheless, administering antimicrobial, amoxicillin/clavulanic acid, to the patient led to improvement of his clinical condition and was followed by a decline in the CRP level. Typically, fever and neutrophilia are clinical hallmarks of infection. Absence of classic clinical signs of infection may be expected in clozapine toxicity settings. PMNs are first line of defence against bacterial infection. Neutrophils employ an oxidative enzyme system, the myeloperoxidase (MPO) system, to generate potent bactericidal oxidants including superoxide, hydrogen peroxide, and hypochlorous acid $[5,6]$. CLZ and its metabolite NCLZ have been reported to exhibit a cytotoxic effect, in a concentration-dependent manner, on the PMNs and their myeloid precursors [6]. In this regard, CLZ and NCLZ can be bioactivated in the peripheral blood PMNs by the MPO system to reactive nitrenium ions which are cytotoxic to PMNs. This toxic effect causes acceleration of the PMN physiologic cell death cycle $[6,7]$. Also, clozapine in a concentration-dependent manner has been reported to reduce cold, drugs, and Lipopolysaccharides- (LPS-) induced-body hyperthermia [8-10]. The CLZ body temperature reducing effect is mediated through its 5 HT $1 \mathrm{~A}$ agonistic and 5 HT 2A antagonistic effect which in turn decreases heat production and increase heat loss through cutaneous vasodilatation.

The major metabolite of clozapine in serum is desmethylclozapine. Demethylation of clozapine is mediated mainly by CYP 1A2 (70\%) and to a lesser extent by CYP 3A4 $[3,4,11,12]$. Under normal conditions, serum levels of NCLZ and CLZ are correlated. An NCLZ : CLZ ratio of $>77 \%$ that increases with treatment duration has been reported in the literature $[4,11,13]$. Approximately 2-5 hours after clozapine administration, concentrations of NCLZ may exceed those of clozapine [13]. Earlier reports indicated an association between infection and CLZ toxicity $[3,4]$. The presumed mechanism for this association suggests that infection downregulates CYP $1 \mathrm{~A} 2$ by about $90 \%$ through increase in circulating IL-6, interferon, and TNF- $\alpha[3,4]$. This, in turn, interferes with the normal CYP 1A2 metabolic conversion of CLZ and hence the ratio of NCLZ: CLZ may decrease. The patient in this report has been using omeprazole as a concomitant medication for gastrointestinal complaints. Omeprazole is a CYP $1 \mathrm{~A} 2$ and CYP $3 \mathrm{~A} 4$ inducer that 


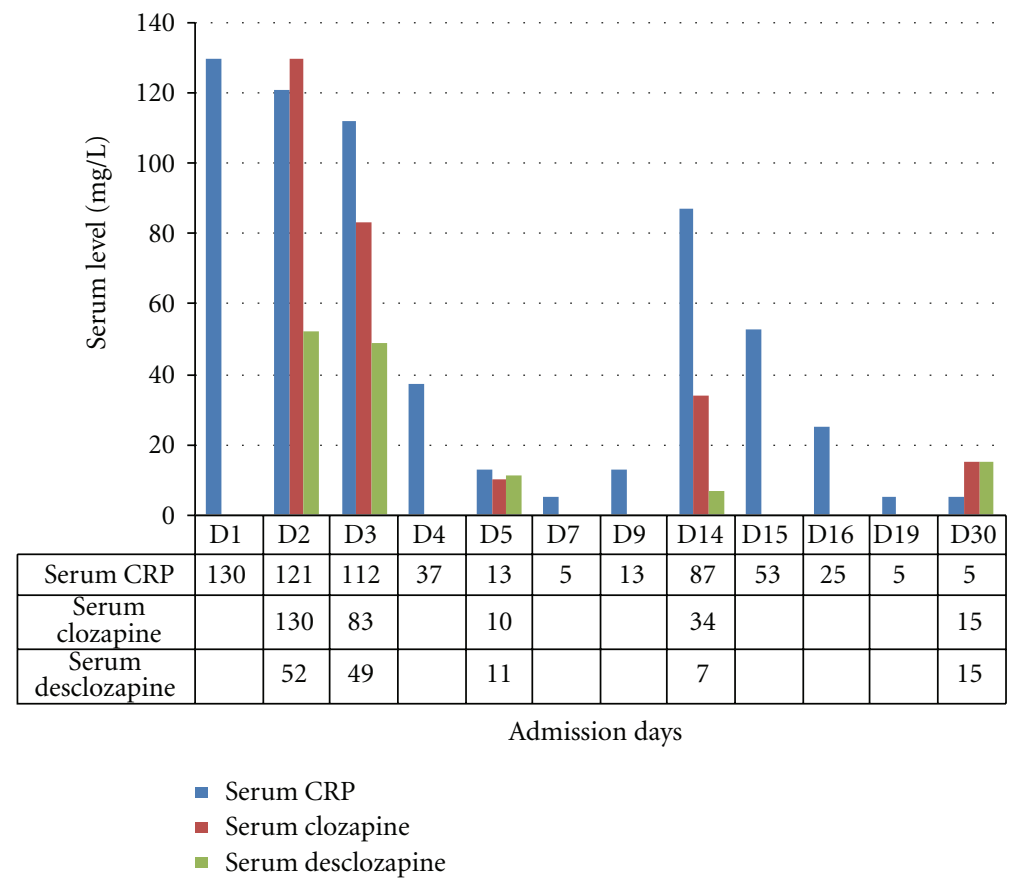

FIGURE 1: Profile of serum CRP (mg/L), clozapine, and norclozapine (measured in $\mu \mathrm{g} / \mathrm{L}$, changed to mg/L and then multiplied by 100 for ease of viewing) levels during admission.

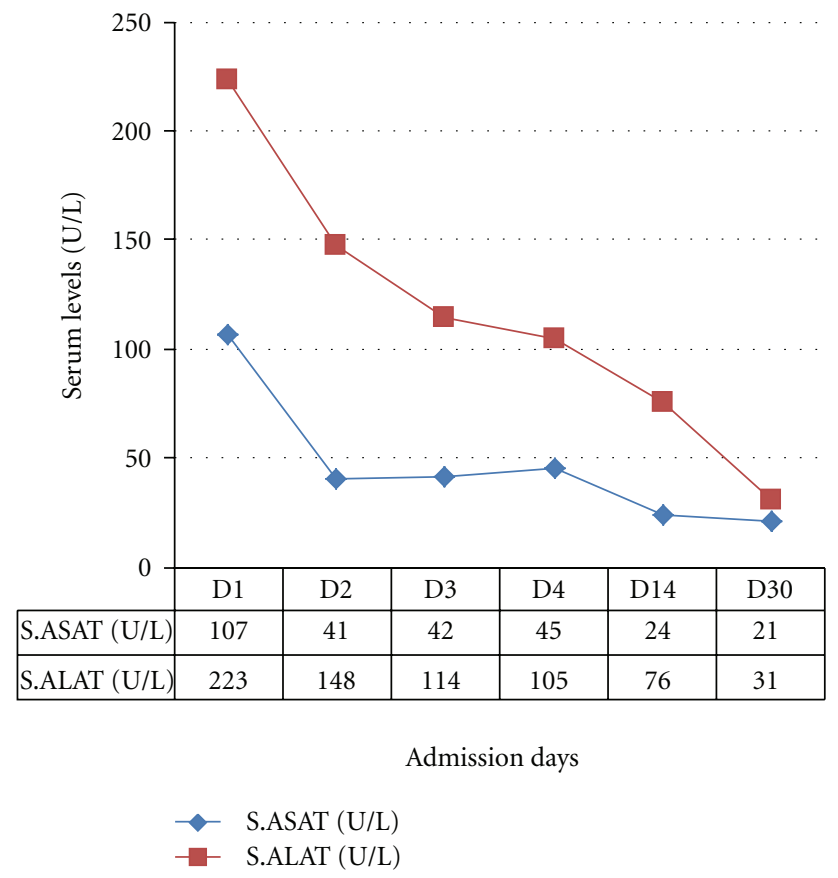

FIgUre 2: Profile of serum transaminases, ASAT and ALAT during admission.

has been reported to reduce CLZ plasma level by $41.9 \%-$ $44.7 \%$ [11]. The observed elevation of CLZ in this patient would, therefore, confirm inhibition of the CLZ metabolizing enzymes and clarify the observed low NCLZ:CLZ ratio (40\%). Meanwhile, infection-induced increases in IL-6 production stimulate hepatocytes to synthesize and secrete acute-phase proteins such as CRP [3]. Taken together, the final result will be a high (toxic) plasma clozapine level, decrease in NCLZ: CLZ ratio, and elevated serum CRP level. Recognition of these laboratory findings could be of crucial importance in psychiatric emergency settings. Finding high toxic clozapine concentration, low NCLZ:CLZ ratio and elevated CRP level, even in the absence of recognizable clinical signs of infection, can be used as an indirect indication of infection-induced clozapine toxicity and hence justify the early use of antimicrobial therapy. 
Another interesting observation is the association between diarrhoea and increased CRP together with decline in the NCLZ: CLZ ratio to $21 \%$ after having reached $107 \%$ on the 5 th admission day, see Figure 1 . The diarrhoea started on the 8th day of amoxicillin/clavulanic acid administration. This may suggest an antibiotic associated diarrhoea (AAD). However, the route of amoxicillin/clavulanic acid administration (i.v.) makes an infectious origin of AAD unlikely and the negative result from the faecal culture excluded diarrhoea of infectious origin. Similarly, an antibioticinduced noninflammatory disturbance in the function of the normal intestinal flora, leading to accumulation of highmolecular-weight carbohydrate in the colon and, therefore, causing osmotic diarrhea [14] cannot explain the elevated CRP and decline in normal CLZ:CLZ ratio. Also, normal serum levels of ASAT (half-life time 12-24 hour) and ALP would argue against recent liver cell injury as a cause of diarrhoea. The observed mild elevation in serum ALAT $(<2$ times ULN) could be explained by the observed high s. ALAT (223 U/L) at admission and the relatively long half-life time of circulatory ALAT (37-57 hour) that resulted in slow decline in serum ALAT concentration. A more conceivable explanation is the early administration of clozapine to a not completely healthy intestinal epithelium. The patient has been under treatment with omeprazole for GIT complaints. Paralytic ileus is associated with bowel compression, which, in turn, leads to irritation and inflammation of the intestinal epithelium [15], and CLZ has been reported to induce colitis and diarrhoea [16]. Taken together, readministration of clozapine to the patient under such conditions might have increased the inflammatory state of the intestinal epithelium leading to diarrhoea and increased secretion of intestinal cytokines including IL-6 $[17,18]$ with elevated CRP and decreased NCLZ: CLZ ratio.

In conclusion, we suggest that an elevated CRP and a reduced NCLZ: CLZ ratio might help in the differential diagnosis between an overdose and an infection/inflammation induced clozapine toxicity and, therefore, help prompt selection of treatment strategy. Further investigations are warranted to confirm this suggestion.

\section{Acknowledgments}

The authors wish to express their appreciation to Mrs. Julie Steenvoorden, Data Manager at the QPS B.V. Netherlands, for revising the linguistic contents of this manuscript. An abstract containing some materials used in this paper was presented at the Dutch society of clinical pharmacology meeting held in March 2012.

\section{References}

[1] "Management of clozapine-induced side effects," Graylands Hospital Drug Bulletin, vol. 13, no. 4, 2005.

[2] P. Pegah Pajouhi and J. A. Bourgeois, "Clozapine, fluoxetine, and benztropine associated ileus: case report," Jefferson Journal of Psychiatry, vol. 21, no. 1, 2007.

[3] P. Darling and M. A. Huthwaite, "Infection-associated clozapine toxicity," Clinical Schizophrenia \& Related Psychoses, vol. 5, no. 3, pp. 159-160, 2011.
[4] E. Wetherby, "Clozapine serum concentration and infectious processes,” BCMHAS Psychopharmacology Newsletter, 2009, http://www.bcmhas.ca/NR/rdonlyres/5893C8EB-5022-456DAF1A-5C110343D708/37163/BCMHAS_Psychopharmacology_Newsletter_Clozapine.pdf

[5] R. W. Bonvillain, R. G. Painter, E. M. Ledet, and G. Wang, "Comparisons of resistance of CF and Non-CF pathogens to hydrogen peroxide and hypochlorous acid oxidants in vitro," BMC Microbiology, vol. 11, article 112, 2011.

[6] D. P. Williams, M. Pirmohamed, D. J. Naisbitt, J. L. Maggs, and B. K. Park, "Neutrophil cytotoxicity of the chemically reactive metabolite(s) of clozapine: possible role in agranulocytosis," Journal of Pharmacology and Experimental Therapeutics, vol. 283, no. 3, pp. 1375-1382, 1997.

[7] D. S. Robinson, "Clozapine agranulocytosis: mechanism of drug toxicity," Primary Psychiatry, vol. 13, no. 3, pp. 27-29, 2006.

[8] W. W. Blessing, "Clozapine and olanzapine, but not haloperidol, reverse cold-induced and lipopolysaccharide-induced cutaneous vasoconstriction," Psychopharmacology, vol. 175, no. 4, pp. 487-493, 2004.

[9] W. W. Blessing, B. Seaman, N. P. Pedersen, and Y. Ootsuka, "Clozapine reverses hyperthermia and sympathetically mediated cutaneous vasoconstriction induced by 3,4methylenedioxymethamphetamine (ecstasy) in rabbits and rats," Journal of Neuroscience, vol. 23, no. 15, pp. 6385-6391, 2003.

[10] Y. Ootsuka and W. W. Blessing, "5-Hydroxytryptamine 1A receptors inhibit cold-induced sympathetically mediated cutaneous vasoconstriction in rabbits," Journal of Physiology, vol. 552, no. 1, pp. 303-314, 2003.

[11] A. Frick, J. Kopitz, and N. Bergemann, "Omeprazole reduces clozapine plasma concentrations: a case report," Pharmacopsychiatry, vol. 36, no. 3, pp. 121-123, 2003.

[12] K. Linnet and O. V. Olesen, "Metabolism of clozapine by cDNA-expressed human cytochrome P450 enzymes," Drug Metabolism and Disposition, vol. 25, no. 12, pp. 1379-1382, 1997.

[13] H. Weigmann, S. Härtter, V. Fischer, N. Dahmen, and C. Hiemke, "Distribution of clozapine and desmethylclozapine between blood and brain in rats," European Neuropsychopharmacology, vol. 9, no. 3, pp. 253-256, 1999.

[14] C. Hogenaur, H. F. Hammer, G. J. Krejes, and E. C. Reisinger, "Mechanism and management of antibiotic-associated diarrhea," Clinical Infectious Diseases, vol. 27, no. 4, pp. 702-710, 1998.

[15] S. Mukherjee, "Ileus," 2011, http://emedicine.medscape.com/ article/178948-overview.

[16] L. Pelizza and M. Melegari, "Clozapine-induced microscopic colitis: a case report and review of the literature," Journal of Clinical Psychopharmacology, vol. 27, no. 6, pp. 571-574, 2007.

[17] S. J. D. Vitkus, A. Hanifan, and D. W. McGEE, "Factors affecting Cocoa-2 intestinal epithelial cell interleukin-6 secretion," In Vitro Cellular \& Developmental Biology, vol. 34, pp. 660664, 1998.

[18] E. C. Reisinger, C. Fritzsche, R. Krause, and G. J. Krejs, "Diarrhea caused by primarily non-gastrointestinal infections," Nature Clinical Practice Gastroenterology and Hepatology, vol. 2, no. 5, pp. 216-222, 2005. 


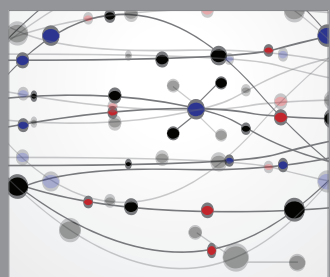

The Scientific World Journal
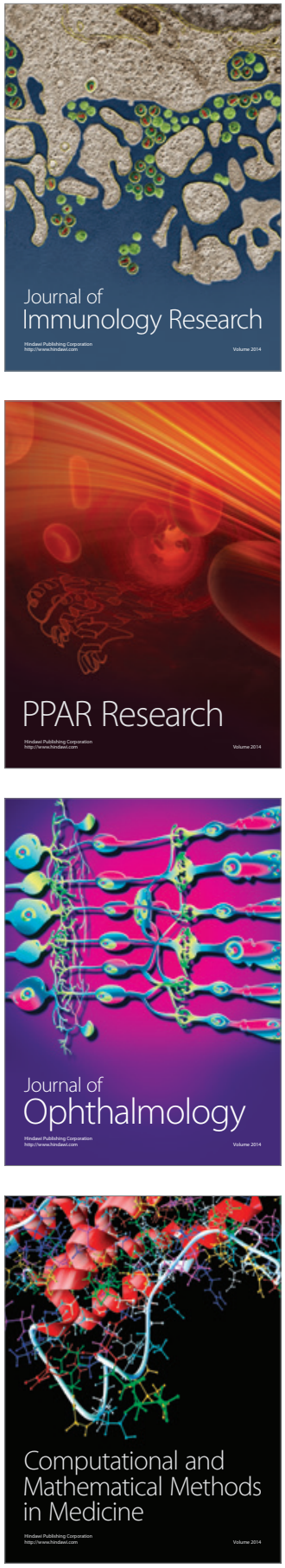

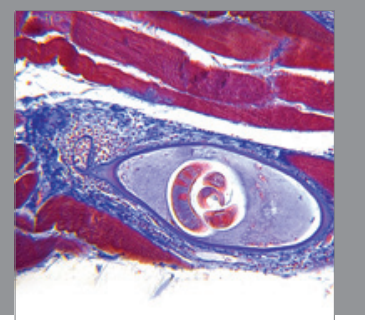

Gastroenterology

Research and Practice
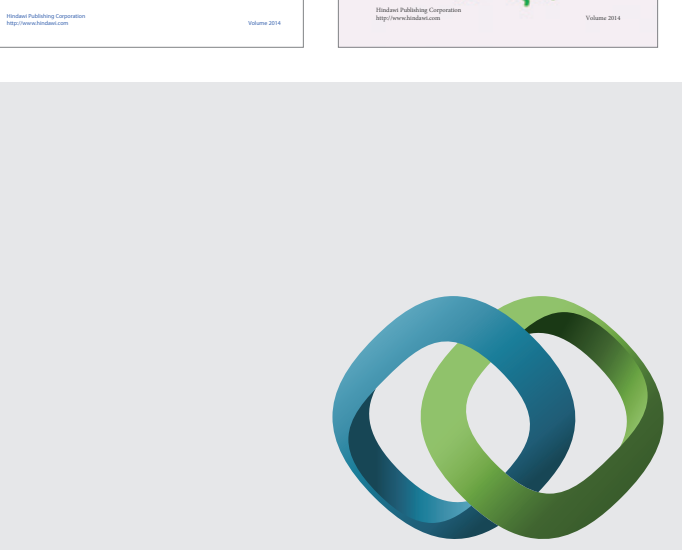

\section{Hindawi}

Submit your manuscripts at

http://www.hindawi.com
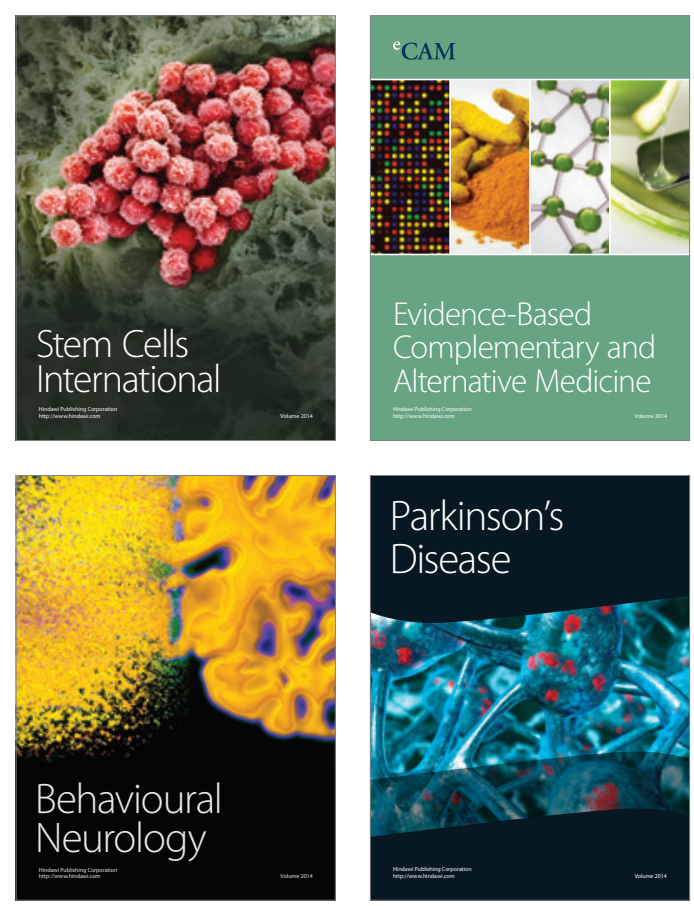

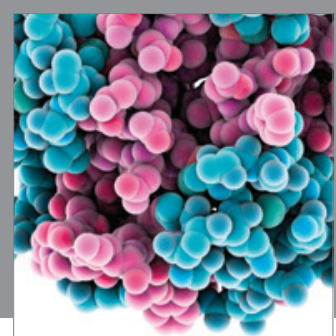

Journal of
Diabetes Research

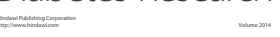

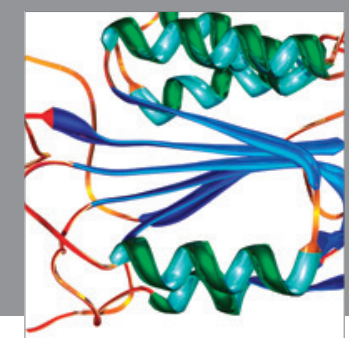

Disease Markers
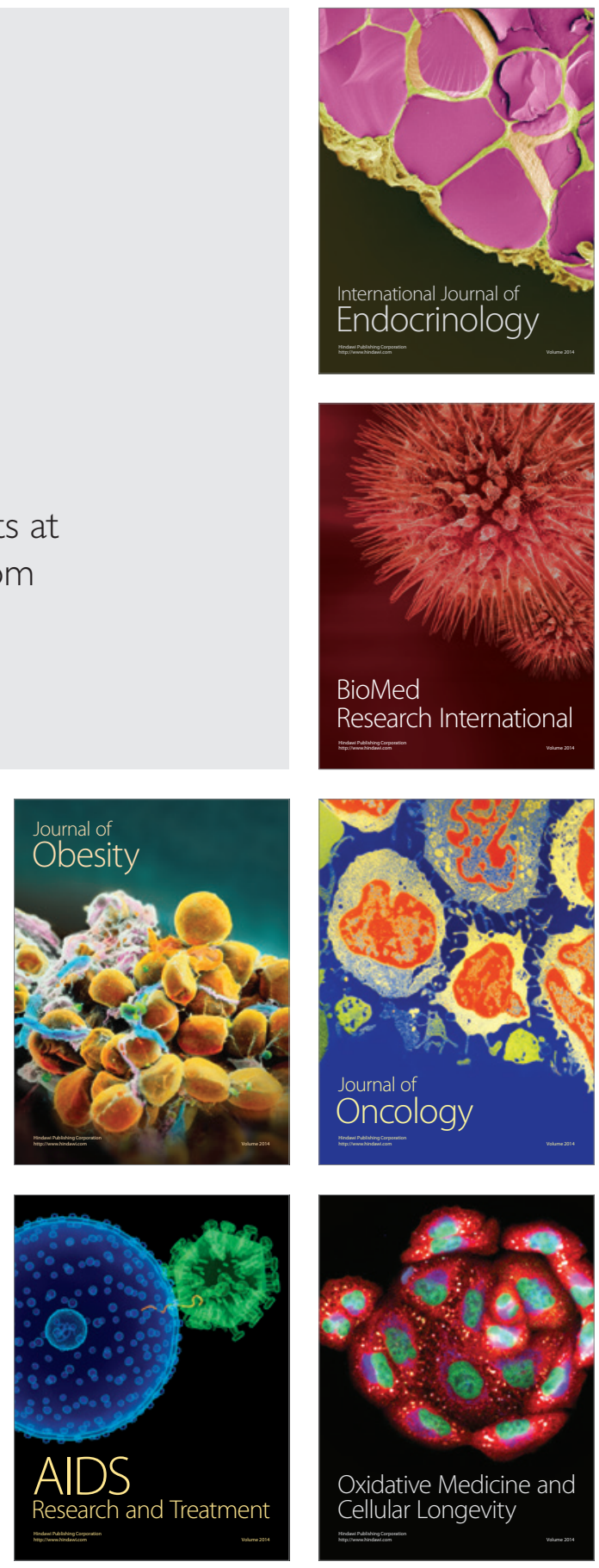